\title{
Écrire la défaite militaire en pays bourguignon. Pour une réévaluation du discours sur la guerre
}

\author{
Christophe MASSON \\ Université de Liège - Transitions. Unité de recherche \\ sur le Moyen Âge tardif \& la première Modernité
}

De l'histoire des entreprises militaires bourguignonnes, on retient certainement tout autant les grandes victoires, comme Othée ou Gavre, que les défaites sanglantes, telles Nicopolis ou Nancy. Et, dans les deux cas, on s'attache avant tout à reconstituer la marche des événements, grâce à une multitude de sources, et donc à éliminer les textes qui dissimuleraient la vérité. Pourtant, c'est aussi dans ces derniers que se dessine en filigrane le portrait de la société militaire bourguignonne, sous la plume, entre autres, des vétérans des guerres ducales. Nous nous consacrerons dans les pages qui vont suivre à tenter de découvrir ce que nous dit le discours sur la défaite, au-delà des accommodements avec la façon dont les événements «se sont vraiment passés »

Pour aborder cette vaste thématique, nous nous concentrerons ici sur deux Mémoires, rédigés par Jean de Haynin et Olivier de la Marche, qui tous deux connurent, entre autres, les victoires et les défaites du duc Charles si bien étudiées par le dédicataire du présent volume ${ }^{1}$. Comment donc ont-ils dit ou camouflé la défaite tactique ${ }^{2}$ ? Quels sont les points qu'ils jugent dignes d'attention, ainsi que ceux qu'ils passent éventuellement sous silence ?

Plusieurs cas de figure se présentent, reflets des accidents des campagnes des armées bourguignonnes. En premier lieu vient la défaite que l'on ne cherche pas à cacher. À l'exemple de la bataille d'Arras du 27 juin 1475, il est des circonstances où l'on ne veut, ou ne peut, dissimuler l'issue des combats. Jean de Haynin expose les événements de façon très technique, soulignant combien le fait d'avoir été pris à revers oblige les Bourguignons, malgré leur vaillance qui leur permet de repousser l'avant-garde française, à se retirer du champ de bataille ${ }^{3}$. La Marche en fait de même avec Grandson ${ }^{4}$. À côté de l'attachement à la relation d'une certaine vérité, il faut insister ici sur le fatalisme des combattants du Moyen Âge face aux aléas de Fortune. Ainsi eust le duc de Bourgoingne la fortune deux fois contre luy en peu de tems dit d'ailleurs Olivier à propos de Grandson et Morat ${ }^{5}$. Cette crainte du hasard est largement diffusée dans la société, comme l'illustrent les auteurs de traités d'art militaire qui déconseillent d'affronter l'adversaire en champ ouvert, car le hasard a une trop grande part dans la décision finale, et recommandent plutôt les coups de main d'envergure plus réduite, offrant de meilleures chances de succès ${ }^{6}$.

1 Que celui-ci veuille bien voir dans ce choix un hommage à ses travaux, dont bien entendu son livre Louis XI et Charles le Hardi. De Péronne à Nancy (1468-1477) : le conflit, Bruxelles, 1996 dans le contexte duquel s'ancre cet article, et à l'amitié qu'il nous témoigne depuis plusieurs années.

2 Dans le cadre de cet article, pour surmonter la difficulté de déterminer, pour tout affrontement, un vainqueur et un vaincu et puisque c'est la réaction des combattants face à la situation sur le champ de bataille, nous ne qualifierons de défaite que les échecs tactiques, et non diplomatiques, politiques ou sociaux. Voir à ce sujet J.-M. LE GALL, Les défaites à la Renaissance, ou l'impossible traumatisme, dans La défaite à la Renaissance, éd. ID., Genève, 2016, p. $26-30$.

3 Jean de Haynin, Mémoires. 1465-1477, éd. D. D. Brouwers, t. II, Liège, 1906, p. 201-202.

4 Olivier de la Marche, Mémoires, éd. H. Beaune, J. D'Arbaumont, t. I, Paris, 1883, p. 139-140.

5 Ibid., t. III, Paris, 1885, p. 211 (nous soulignons).

6 V. par ex. Le livre de l'art de chevalerie de Végèce. Trad. anonyme de 1380, éd. L. LöFSTEDT et alii, Helsinki, 1989, p. 91, 99-101 ou ANTOINE DE LA SALE, La Salade, dans ID., Euvres complètes, éd. F. DeSONAY, t. I, Liège-Paris, 1935, p. 243. 
La Fortune, cependant, ne fait pas tout. Elle trouve une rivale acharnée dans la Vertu ${ }^{7}$ des capitaines bourguignons. Nos deux auteurs ont ainsi cherché à souligner ce qui, malgré la défaite de leur camp, la rendait moins déplorable et critiquable. À Morat et Nancy, c'est l'infériorité numérique qui est la cause de la défaite ${ }^{8}$. Le capitaine n'a donc pas failli dans la conduite de ses hommes ${ }^{9}$. L'échec de l'assaut dirigé contre la ville de Huy en 1467, sur la route de la bataille de Brusthem, est imputé non aux hommes mais au manque d'effectifs - la troupe du seigneur de Ravenstein ne vient pas soutenir l'effort de la compagnie du sire de Fiennes. Haynin insiste ensuite sur la grande réussite que représente la retraite en bon ordre qui conduisit les troupes bourguignonnes jusqu'en terre namuroise. Alors que les chemins sont mauvais et les bois boueux, ce qui rend toute l'entreprise périlleuse et compliquée, il sanbla a pluseurs que che fut une belle aventure den estre retourne a si peu de perte ${ }^{10}$. Cette attention témoignée aux résultats humains des combats - la mort, la blessure, l'emprisonnement mais aussi la protection et la bonne conduite - est une constante qui semble dépasser, ou pour mieux dire amender, les résultats du champ de bataille. Les campagnes de France du début des années 1470 en donnent de beaux exemples. Si les Français y font la démonstration d'une nette supériorité tactique et emportent toute une série de places dans la Somme, les Bourguignons n'ont rien à leur envier sur le plan de la valeur militaire, s'il faut en croire Jean de Haynin. Celui-ci dépeint les combats de la tour de Saint-Souplet en 1471 avec un pinceau tout entier gagné à la cause de son châtelain bourguignon, Collart Dencourt. C'est à lui qu'est due la résistance au premier assaut français, largement décrite. Il s'agit là d'un préalable à la retraite bourguignonne, conduite sans combat dans la crainte d'un retour français et brièvement évoquée. De la sorte ressort non l'échec de l'homme de guerre incapable de défendre les terres de son seigneur mais bien la réussite qu'a été sa façon d'évacuer ses troupes afin de les sauver d'un second affrontement, sans aucun doute tout aussi sanglant que le premier. Si cet accrochage était bien mineur, on ne pouvait en dire autant de l'assaut de Beauvais, échec si pas stratégique à tout le moins personnel du Téméraire. Mais là encore, par le biais d'une lettre d'un vétéran des combats incluse dans son texte, Haynin donne à voir à son lecteur combien cette retraite fut la plus belle de james et la plus fierre; car nous partimes par establyes leun apres lautre de tou costés, tous de troix conpagnies, les deux le visage tournés vers eulx tandi que la tierche marchoit, contrebalançant de la sorte sa relation de l'échec ${ }^{11}$. La discipline militaire, et en dernier ressort les qualités du capitaine ${ }^{12}$, viennent ici racheter la défaite.

Enfin, la dernière étape de ce discours est, si l'on peut dire, la défaite transformée en victoire. Monthléry ressortit, tout du moins en partie, à cette catégorie. Jean de Haynin fait la balance des événements. D'un côté il reconnaît que les Français s'imposèrent du point de vue du nombre de prisonniers faits au cours de la journée. Il en donne d'ailleurs une longue explication et insiste, en ouverture de son récit, sur un chevallier portant ung ghidon des archies d'aucun des cappitaines princhipaus et oussi ung des gran chies de chanbre de toute l'armée, et pluseurs autres chevalliers, gentis hommes, homes d'armes et archies, tant de cors come autres, [qui] s'en fuyrte

7 B. STERCHI, The Importance of Reputation in the Theory and Practice of Burgundian Chivalry. Jean de Lannoy, The Croÿs, and the Order of the Golden Fleece, dans The Ideology of Burgundy. The promotion of National Consciousness. 1364-1565, éd. D.J.D. Boulton, J.R. VeEnSTRA, Leyde-Boston, 2006, p. 101 n. 12.

8 JeAn de Haynin, Mémoires, t. II, op. cit., p. 220, 224 ; Olivier DE LA MARCHe, Mémoires, t. I, op. cit., p. 140, 142 ; t. III, op. cit., p. 240. Ce dernier (Ibid., p. 77, 79) explique de la même façon l'échec devant Beauvais. M. DEPRETER, Moult cruaultéz et inhumanitéz y furent faictes. Stratégie, justice et propagande de guerre sous Charles de Bourgogne (1465-1477), dans Le Moyen Âge, t. CXXI/I, Le pouvoir par les armes, le pouvoir par les idées, éd. J. DuMONT, C. MASSON, 2015, p. 64-65, 69, explique comment les perspectives de la défaite, et de la rigoureuse sanction qui devait s'ensuivre, ont poussé les assiégés à résister aux Bourguignons.

9 La mention de JEAN DE HAYNIn, Mémoires, t. II, op. cit., p. 219 selon laquelle la défaite de Grandson est imputable à une erreur tactique du duc Charles n'est, selon son éditeur, pas de la main de cet auteur (n. 1).

10 JeAn DE HaYnin, Mémoires. 1465-1477, éd. D. D. BrouWERs, t. I, Liège, 1905, p. 220-221 (citation).

11 JEAN DE HAYNIN, Mémoires, t. II, op. cit., p. 113, 136, 142 (citation).

12 J. DevauX, L'image du chef de guerre dans les sources littéraires, dans Publication du Centre européen d'Études bourguignonnes (XIV $-X V I^{e}$ s.), t. XXXVII, Rencontres de Nivelles-Bruxelles, 26-29 septembre 1996. Images et représentations princières et nobiliaires dans les Pays-Bas bourguignons et quelques régions voisines, 1997, p. 129. 
honteusement et lesserte et abandonerte leur prinche et leur cappitaine [Charles, alors comte de Charolais $]^{13}$. La Marche n'est pas en reste qui rappelle que s'enfuirent aucungs des cappitaines bourguignons, dont la compaignie du conte [de Charolais] fut fort amoindrie. Il conclut d'ailleurs, cruellement laconique : Je ne les scauroye comment excuser ${ }^{14}$. Mais tous deux sont unanimes, la victoire fut bourguignonne. Les règles de la guerre leur permettent en effet d'affirmer que Charles triompha le 16 juillet 1465 , puisqu'il tenait le champ au soir du combat ${ }^{15}$. Les Français ont beau avoir fait plus de butin, matériel et humain, que leur adversaire, le triomphe leur a échappé. Le siège de Neuss est à ranger dans la même catégorie. Pour Jean de Haynin, reprenant une lettre du Hardi destinée au comte de Chimay, c'est une victoire des troupes bourguignonnes sur l'armée mobilisée par l'empereur Frédéric III de Habsbourg qui permet l'ouverture de négociations entre les belligérants, négociations au cours desquelles l'artillerie et les prisonniers bourguignons seront rendus à leur camp ${ }^{16}$. Plus loin dans son texte Haynin, évoquant à nouveau les opérations menées au cours du siège de Neuss, soulignera que la rencontre entre Bourguignons et Impériaux n'a pas livré de verdict bien net, mais que les Bourguignons perdirent moins d'hommes que leurs adversaires au terme d'une journée qui vit les deux camps contraints à la retraite ${ }^{17}$. Il reprend donc la hiérarchisation des signes de victoire déjà évoquée à l'occasion de la bataille de Montlhéry. Personne ne tenant le champ, le regard se porte sur l'issue humaine du combat. Les Bourguignons ayant infligé plus de pertes qu'ils n'en subirent, ils ne pouvaient qu'être les vainqueurs. Or, sur le terrain, cet affrontement fut plutôt une réussite impériale, puisque à sa suite Frédéric III parvient à renforcer la position de ses troupes dans la région ${ }^{18}$. Quant à la négociation, elle permettra surtout à ce dernier de s'assurer du retrait de Charles. Une dernière étape est même franchie lorsque Olivier de la Marche fait du duc de Bourgogne un quasi démiurge, capable de

grans choses dignes de memoire. Car il tint le siege devant ladicte ville ung an entier; il destourna rivieres de leurs cours ; il dicqua ung bras du Rhin; il gaigna ung isle et par les dicques il venoit à pied secq ; il fist faire chaz, grues et aultres engins, comme trenchis, rollans, bastillons et toutes maneres dont on peut villes approchier. Grans battures furent faictes, grans essays et assaulx, et jusques à faire essais de nagier le Rin à cheval, la lance sur la cuisse ${ }^{19}$.

Le pouvoir du duc de Bourgogne ne connaît donc plus de limite terrestre, ses actions militaires semblant faire sur les vétérans une impression aussi forte que la «ville de bois et de toile » qu'on érigea devant la ville eut sur les ambassadeurs qui s'y relayèrent inlassablement ${ }^{20}$. De limite, au final, il n'y en eut qu'une, et de taille, la résistance des Colonais.

Les publics d'Olivier de la Marche et de Jean de Haynin, s'ils n'étaient peut-être pas identiques, n'en ressortissaient pas moins au même univers. Au sommet de ce dernier trônait le prince Philippe, à qui s'adressait directement le premier d'entre eux. Ensuite venaient les indiciaires,

13 JEAN DE HAYNIN, Mémoires, t. I, op. cit., p. 68 (citation), 77.

14 OliviER DE LA MARCHE, Mémoires, t. III, op. cit., p. 12.

15 Jean de Haynin, Mémoires, t. I, op. cit., p. 77 ; Olivier de la Marche, Mémoires, t. I, op. cit., p. 124 ; t. III, op. cit., p. 17-18.

16 Jean de Haynin, Mémoires, t. II, op. cit., p. 187 ; Olivier De la MarChe, Mémoires, t. I, op. cit., p. 138 ; t. III, op. cit., p. 100 - dont la relation des événements ne pouvait que faire écho à la situation de Philippe le Beau, destinataire de ses Mémoires, qui voyait converger en lui les intérêts communs des Valois de Bourgogne et des Habsbourg - fait, au moyen d'une ellipse, du combat livré en mai la cause de la négociation et du départ des belligérants qui prend place en juin.

17 JEAN DE HAYNIN, Mémoires, t. II, op. cit., p. 199, reprend ici la teneur d'un courrier envoyé par le comte de Chimay à son épouse. Cet auteur semble faire deux combats différents de l'affrontement qui eut lieu en mai 1475.

18 J.-M. CAUChIES, Louis XI et Charles le Hardi, op. cit., p. 93 ; R. VAughan, Charles the Bold. The Last Valois Duke of Burgundy, $\mathrm{n}^{\text {le }}$ éd., Woodbridge, 2002, p. 342-343.

19 OliviER DE LA MARChe, Mémoires, t. I, op. cit., p. 136-137.

20 J.-M. CAUCHIES, Charles le Hardi à Neuss (1474/75) : folie militaire ou contrainte politique ?, dans Publication du Centre européen d'études bourguignonnes (XVe-XVI s.), t. XXXVI, Rencontres de Nimègue (21 au 24 septembre 1995). Pays bourguignons et terres d'Empire (XVe-XVI s.) : rapports politiques et institutionnels, 1996, p. $108-109$. 
qui semblent constituer l'auditoire tant du $\operatorname{premier}^{21}$ que, en toute hypothèse, du second ${ }^{22}$. On pourrait donc voir dans leurs traitements de la défaite, qui se répondent parfaitement, une volonté d'introduire chez les historiographes des référents culturels propres aux hommes de guerre, produits en même temps que composants de la société militaire bourguignonne. Et donc de veiller au portrait que les vecteurs officiels de l'histoire bourguignonne pourrait dresser de l'armée ducale. Et si même, dans le cas de Haynin, la transmission du seul manuscrit complet de son œuvre au sein de sa famille $^{23}$ indiquerait que là était son public désigné, l'interprétation des choses peut ne point changer, le vétéran des guerres bourguignonnes exposant à ceux qui lui succéderont les qualités de la troupe qu'ils intégreraient bientôt. Le mémorialiste est donc bien un "formateur [qui] fait partager à son public une expérience qu'il lui prodigue sans restriction morale ni édifiante ${ }^{24} »$. Pour ces vétérans du métier des armes que sont Jean et Olivier, et c'est là l'idée qu'ils veulent transmettre à leurs lecteurs, la défaite est le fait de tous et de personne à la fois, elle ne s'accompagne pas d'un blâme sur la société militaire dans son ensemble. Celle-ci se trouve même glorifiée d'avoir résisté à la défaite, de ne pas s'être décomposée sous les coups de boutoirs des ennemis. Le portrait que trace la défaite dans les Mémoires de Haynin et de la Marche est avant tout celui d'une société de frères d'armes, solidaire, une sorte de havre des valeurs morales les plus excellentes, celles de la chevalerie qui se diffusent loin au-delà de la seule aristocratie, loin au-delà des seuls adoubés ${ }^{25}$. Il n'est donc pas question de nier les revers de fortune rencontrés par les armées mais bien de leur donner une explication en même temps que de célébrer le courage des hommes d'armes qui paraît s'imposer comme la première des valeurs chevaleresques ${ }^{26}$. Si la volonté d'anoblir l'armée, ou plus exactement ses membres, n'est pas présente dans les démonstrations des deux auteurs ${ }^{27}$, ceux-ci ne leur attribuent pas moins, incidemment, des qualités qui sont propres à l'aristocratie. Le métier des armes n'anoblit pas de facto mais ouvre déjà la voie de l'élévation sociale à ceux qui l'exercent. La noblesse héréditaire cède ici le pas à une noblesse morale, conquise par la seule force de la vertu, que légitiment non les généalogies mais le regard des contemporains ${ }^{28}$. Une identité militaire est en phase de constitution ${ }^{29}$, qui se construit en large part sur la morale chevaleresque. La carrière d'un seul homme, l'auteur des Mémoires en l'occurrence, se trouve dépassée et englobée, sans pour

21 Olivier de la MARChe, Mémoires, t. I, op. cit., p. 185.

22 A.C. DE NĖve DE Roden, Les Mémoires de Jean de Haynin : des " mémoires 》, un livre, dans "A l'heure encore de mon escrire ». Aspects de la littérature de Bourgogne sous Philippe le Bon et Charles le Téméraire, éd. C. THIRY, Louvain, 1997, p. 43-52, développant une idée déjà présente chez C. THIRY, Jean de Haynin, dans Grundriss der romanischen Literaturen des Mittelalters, t. XI/II, La littérature historiographique des origines à 1500 (Partie ducomentaire), éd. H.U. GuMBrecht, D. TILlMANN-BARTYLla, Heidelberg, 1993, p. 143-144.

23 A.C. DE NÈVE DE Roden, Les Mémoires de Jean de Haynin, op. cit., p. 43. Notons qu'outre l'original, quatre copies, abrégées ou très largement postérieures, en ont été identifiées. J. VAN DEN GHEYN, « Le manuscrit original des mémoires du sire de Haynin », Compte rendu des séances de la Commission royale d'histoire, t. LXX, 1901, p. 46.

24 J. BlanchARD, Nouvelle histoire, nouveaux publics : les mémoires à la fin du Moyen Âge, dans L'histoire et les nouveaux publics dans l'Europe médiévale, XIII-XVe siècles. Actes du colloque international organisé par la Fondation européenne de la Science à la Casa de Velasquez, Madrid, 23-24 avril 1993, éd. J.P. GENET, Paris, 1997, p. 41.

25 Ce qui vient confirmer le repérage, par R. W. KAEUPER, Literature as Essential Evidence for Understanding Chivalry, dans Journal of Medieval Military History, t. V, 2007, p. 9, d'un réel esprit de corps parmi la société chevaleresque et, ici, militaire du Moyen Âge.

26 S. DeRuElle, De papier, de fer et de sang. Chevaliers et chevalerie à l'épreuve de la modernité (ca. 1460-ca. 1620), Paris, 2015, p. 110-111.

27 La Marche l'affirmera clairement dans son Livre de l'advis de gaige de bataille, éd. B. Prost, Traités du duel judiciaire. Relations de pas d'armes et tournois par Olivier de La Marche, Jean de Villiers, seigneur de l'Isle-Adam, Hardouin de La Jaille, Antoine de La Sale, etc, Paris, 1872, p. 45.

28 B. STERCHI, The Importance of Reputation, op. cit., p. 101. V. aussi sur ce sujet C. T. ALLMAND, Changing Views of the Soldier in Late Medieval France, dans Guerre et société en France, en Angleterre et en Bourgogne. XIV $X V^{e}$ siècle, éd. P. Contamine, C. Giry-Deloison, M. H. KeEn, Lille, 1991, p. 180. Au vrai, le point de mire constitué par la noblesse se retrouve dans la société bien au-delà de la «classe» militaire. Voir F. AUTRAND, L'image de la noblesse en France à la fin du Moyen Âge. Tradition et nouveauté, dans Comptes rendus des séances de l'Académie des inscriptions et belles-lettres, t. CXXIII/II, 1979, p. 340-354.

29 P. CONTAMINE, «Le combattant dans l'Occident médiéval », Le combattant au Moyen Âge, Actes du $18^{e}$ congrès de la Société des historiens médiévistes de l'enseignement supérieur public, Montpellier, 1987, p. 21. 
autant y disparaître, dans une société plus large, à laquelle sa plume est en quelque sorte une porte d'accès.

Il y a sans doute plus encore. Jean achève ses Mémoires au soir de la défaite de $\mathrm{Nancy}^{30}$, soit au moment où la force militaire bourguignonne n'a jamais été aussi mal considérée. Olivier illustre à l'attention du jeune Philippe de Habsbourg les vertus de la Maison de Bourgogne ${ }^{31}$. Il leur importait à tous deux de célébrer la valeur des armées ducales, grandes dans la victoire - Jean a d'ailleurs commencé à composer le manuscrit que l'on connaît aujourd'hui en 1466, au moment des succès des guerres de Liège ${ }^{32}$ - comme dans la défaite. Peut-être, aussi, était-ce une façon de régénérer l'armée bourguignonne à laquelle on allait encore demander tellement... L'accident qu'est la défaite, comme au vrai la victoire, ne doit pas obérer les qualités propres aux gens d'armes bourguignons. Les deux peuvent coexister, la valeur morale, et par voie de conséquence martiale, n'étant pas déterminée par la seule victoire au sens tactique du terme. La défaite n'existe donc pas que pour elle-même, pour la réalité tactique qu'elle représente. Qu'elle soit sans appel ou contestée, elle n'en permet pas moins de réfléchir aux devoirs des hommes d'armes bourguignons. Elle est l'occasion pour les deux auteurs d'élargir le propos, d'embrasser la guerre de «plus haut», d'en tirer une leçon ${ }^{33}$. Ceux-ci dépassent l'observation " au ras du sol» chère à John Keegan ${ }^{34}$ pour se diriger vers une systématisation de leurs observations militaires. Enseigner des solutions à des problèmes donnés comme la défaite a beau ne pas être le premier objectif des Mémoires, les exemples qu'ils fournissent à leurs lecteurs ne pouvaient que les instruire, de la même façon que devaient le faire les chroniques, les romans de chevalerie ou les manuels didactiques ${ }^{35}$, et les pousser à se montrer aussi bons, voire meilleurs, que leurs prédécesseurs, par la seule vertu de l'émulation. La visée, non seulement historique mais aussi argumentative ${ }^{36}$, contribue à dessiner, ou plus exactement à compléter au gré de variations sur un même thème, le stéréotype du combattant bourguignon ${ }^{37}$. Celui-ci n'est pas uniquement le preux qui par la seule force de son bras abat le capitaine ou le roi adverse mais devient également le sage qui, dans la défaite, prévient la catastrophe. Et, par l'habitude que prennent Haynin et la Marche de ne pas attribuer ce mérite au seul capitaine, ils laissent entendre que ces deux qualités ne lui sont pas réservées ${ }^{38}$.

30 Jean de Haynin, Mémoires, t. I, op. cit., p. VI ; A.C. DE NÈVE DE Roden, Les Mémoires de Jean de Haynin, op. cit., p. 32.

31 J. Devaux, Le culte du héros chevaleresque dans les Mémoires d'Olivier de la Marche, dans Publication du Centre européen d'études bourguignonnes (XIV $-X V I^{e}$ s.), t. XLI, Rencontres d'Édimbourg-Glasgow (28 septembre au $1^{\text {er }}$ octobre 2000). Le héros bourguignon : histoire et épopée, 2001, p. 58. La première rédaction débutant en 14721473 (C. EMERSON, Olivier de La Marche and the Rhetoric of Fifteenth-Century Historiography, Woodbridge, 2004, p. 11, 43), son contexte est, comme dans le cas de Jean de Haynin, marqué par une période d'activité militaire et de remise en question des qualités de l'armée et du duc bourguignons.

32 A.-C. DE NÈve DE Roden, Les Mémoires de Jean de Haynin, op. cit., p. 33.

33 C'est d'ailleurs l'ambition générale que leur reconnaît J. DUFOURNET, Commynes et l'invention d'un nouveau genre historique : les mémoires, dans Chroniques nationales et chroniques universelles. Actes du colloque d'Amiens, 1617 janvier 1988, éd. D. BusCHINGER, Göppingen, 1990, p. 71-73, en signalant toutefois les limites des deux auteurs en ce domaine.

34 Cette volonté des auteurs de préférer à leur seul point de vue une vision explicative des événements a été mise en évidence par Y.N. HARARI, Renaissance Military Memoirs. War, History and Identity, 1450-1600, Woodbridge, 2004 , e.a. p. 85.

35 S. FOURCADE, De l'utilité des lettres dans la carrière des armes. Guerre et culture écrite en France au XV siècle, dans Le Moyen Âge, t. CXXI/I, op. cit., p. 21-40.

36 Ce que N. KUPERTY-Tsur, Se dire à la Renaissance. Les Mémoires au XVI siècle, Paris, 1997, p. 195, observe pour la carrière des auteurs du XVI ${ }^{\mathrm{e}}$ siècle - qui ont à la défendre - se retrouve ici dans le cadre plus large de la société militaire.

37 Cet attachement au stéréotype du chevalier - qu'il ne nous appartient pas ici de démontrer moins anachronique qu'il n'est souvent présenté - de la part d'Olivier de la Marche a déjà été mis en évidence par H. WOLFF, La caractérisation des personnages dans les Mémoires d'Olivier de la Marche : Identification ou description?, dans Revue des langues romanes, t. XCVII/I, Écrire l'histoire à la fin du Moyen Âge, éd. J. DuFOURNET, L. DULAC, 1993, p. 43 et peut être appliqué à Jean de Haynin.

38 Ils ouvrent donc la porte à l'assimilation des hommes d'armes au portrait que les auteurs de romans font, à la même époque, du chevalier idéal qui doit «allier en lui prouesse et sagesse». D. QUERRUEL, Du chevalier au 
En conclusion, il ressort de cette enquête préliminaire, qui devrait être élargie aux Mémoires français ainsi qu'aux biographies consacrées à des chevaliers bourguignons et français, à l'image des Livre des fais de Jacques de Lalaing ou de Jean II Le Meingre, dit Boucicaut, que, à l'image d'autres thématiques, l'histoire de la guerre ne peut pas se résumer à une analyse mathématique. Il y a, dans le discours qui lui est consacré, une ligne de force qui s'organise non seulement autour de qui a remporté la décision sur le champ de bataille mais aussi autour des valeurs propres à l'action martiale. Présenter une défaite comme une victoire ne revient pas uniquement à maquiller la vérité mais plutôt, car il était probable que les lecteurs savaient bien qu'on leur «mentait », à souligner ce qui doit servir d'exemple. En cela, la Marche et Haynin - pour qui il s'agit là du seul domaine où il nous livre un avis original ${ }^{39}$ - tirent profit de leur expérience d'homme de guerre pour construire un système plus large. Les combattants, capitaines ou subordonnés, qui assurent un retrait sans accroc sur le champ de bataille sont les clefs de voûte d'un système plus large. Ainsi sans doute est-il, aux yeux des deux auteurs, plus important de se comporter comme un digne et valeureux homme d'armes que de remporter la bataille à une occasion précise. Les ducs de Bourgogne, et à leur suite Maximilien de Habsbourg, le propre père de Philippe le Beau, s'étaient d'ailleurs toujours attachés à remplir leur devoir militaire de la meilleure des façons ${ }^{40}$. Le capitaine valeureux dans la victoire comme dans la défaite est donc à la fois le rappel des qualités martiales des ducs, de leur devoir comme responsables, sur le champ de bataille, de leurs hommes, d'une part, et une métaphore, bien que plus esquissée que développée, des devoirs du duc vis-à-vis de ses sujets, d'autre part. Le discours sur l'armée et ses membres décrit un idéal état d'équilibre ${ }^{41}$ où ne peuvent exister les insuffisances des gens d'armes, même si le duc est alors occupé à réorganiser son armée. Ce n'est d'ailleurs pas une surprise que ces Mémoires aient été rédigés simultanément, ou presque, aux réformes du Téméraire. Le moment était manifestement favorable à une réflexion sur le fait militaire, même si ce n'était pas là l'objectif premier des deux auteurs.

La défaite, accident de la Fortune, vient en somme compléter le portrait de l'exercice de la guerre. Nos deux auteurs demeurent donc consciemment hors de la culture de la défaite - une culture éminemment politique - pour laquelle l'échec est le signe d'une réforme nécessaire ${ }^{42}$. Cette attitude est sans doute un des ingrédients d'une culture de guerre par laquelle la société militaire se maintient séparée du reste de la société. Le Bourguignon est ainsi en tous lieux un digne chevalier, même si souvent Fortune varie.

"chevetaine ": images de la guerre dans les romans bourguignons du XV siècle, dans Revue des langues romanes, t. CXVII/II, La guerre au Moyen Âge: des motifs épiques aux réalités du XVe siècle (vol. 2), éd. J. DUFOURNET, C. LACHET, 2013, p. 349.

39 A.C. DE NĖVe De Roden, Les Mémoires de Jean de Haynin, op. cit., p. 40.

40 Voir ainsi B. SCHNERB, L'État bourguignon. 1363-1477, Paris, 1999, p. 262

41 Les remarques d'A. THIÉBLEMONT, Les apports de la recherche historique à la compréhension de la société militaire contemporaine, dans Histoire militaire et sciences humaines, éd. L. HENNINGER, Bruxelles, 1999, p. 32-33, sur le fantasmé "état d'équilibre » auquel aspirent les auteurs militaires contemporains sont transposables au contexte médiéval.

42 J.-M. LARgEAUD, «Les cultures de la défaite en Europe et aux Amériques au XIX siècle », Hypothèses, t. XI, 2008, p. 328 ; J.-M. LE GALL, Les défaites à la Renaissance, op. cit., p. 35 ; et, évidemment, W. SCHIVELBUSCH, The Culture of the Defeat. On National Trauma, Mourning and Recovery, Londres, 2003. 